\title{
Label-free optical biosensing with slot-waveguides
}

\author{
Carlos A. Barrios, María José Bañuls, Victoria González-Pedro, Kristinn B. Gylfason, \\ Benito Sánchez, Amadeu Griol, A. Maquieira, H. Sohlström, M. Holgado, and R. Casquel \\ Instituto de Sistemas Optoelectrónicos y Microtecnología, Universidad Politécnica de Madrid, ETSI \\ Telecomunicacion, Ciudad Universitaria s/n, 28040 Madrid, Spain \\ Departamento de Química, Universidad Politécnica de Valencia, Camino de Vera s/n, 46022 Valencia, Spain \\ Microsystem Technology Laboratory, School of Electrical Engineering, KTH - Royal Institute of Technology, \\ Osquldas väg 10, SE-10044 Stockholm, Sweden \\ Centro de Tecnología Nanofotónica, Universidad Politécnica de Valencia, Camino de Vera s/n, \\ 46022 Valencia, Spain \\ Centro Láser, Universidad Politécnica de Madrid, Campus Sur, 28031 Madrid, Spain \\ Corresponding author: cbarrios@die.upm.es
}

\begin{abstract}
We demonstrate label-free molecule detection by using an integrated biosensor based on a $\mathrm{Si}_{3} \mathrm{~N}_{4} / \mathrm{SiO}_{2}$ slotwaveguide microring resonator. Bovine serum albumin (BSA) and anti-BSA molecular binding events on the sensor surface are monitored through the measurement of resonant wavelength shifts with varying biomolecule concentrations. The biosensor exhibited sensitivities of 1.8 and $3.2 \mathrm{~nm} /\left(\mathrm{ng} / \mathrm{mm}^{2}\right)$ for the detection of anti-BSA and BSA, respectively. The estimated detection limits are 28 and $16 \mathrm{pg} / \mathrm{mm}^{2}$ for anti-BSA and BSA, respectively, limited by wavelength resolution.
\end{abstract}

Label-free biomolecule optical sensing technologies are of great interest because of their flexibility to analyze biomolecular interactions without using fluorescence, absorptive, or radio-labels. This simplifies the assay and allows time-resolved study of the kinetics of biomolecular interactions. Integrated photonic devices used as biosensors present important advantages, such as high sensitivity, small size, and high scale integration. Thus, label-free integrated optical biosensors based on Mach-Zehnder interferometers, directional couplers, microring , and disk resonators have been demonstrated to be very sensitive label-free biosensors.

Recently, we have reported an integrated photonic sensor based on a slot-waveguide resonator. This photonic structure takes advantage of the remarkable property of slot-waveguides to provide high optical intensity in a subwavelength-size low refractive index region (slot-region) sandwiched between two high refractive index strips (rails) . This permits a very high interaction between the slot-waveguide mode probe and a liquid analyte. As a result, the reported slot-waveguide sensor exhibited a bulk ambient sensitivity as high as $212.1 \mathrm{~nm} /$ refractive index unit (RIU), which is more than twice as large as that exhibited by ring resonator optical sensors based on conventional strip waveguides. In this Letter we demonstrate the detection of label-free molecular binding reactions on the surface of a slot-waveguide ring resonator. Bovine serum albumin (BSA) protein and anti-BSA are used to study the biosensor performance.

The device consists of a $70 \mu \mathrm{m}$ radius slotwaveguide ring resonator made of $\mathrm{Si}_{3} \mathrm{~N}_{4}$ on $\mathrm{SiO}_{2}$. The $\mathrm{Si}_{3} \mathrm{~N}_{4}$ rails of the slot-waveguide ring are separated by $200 \mathrm{~nm}\left(w_{\text {slot }}\right)$, and their widths are 400 and $550 \mathrm{~nm}$ for the outer and inner rails, respectively, as illustrated in Fig. 1(a). A beam propagation method calculation of the quasi-TE optical mode of the ring slot-waveguide at $1.3 \mu \mathrm{m}$ operation wavelength is

(a)

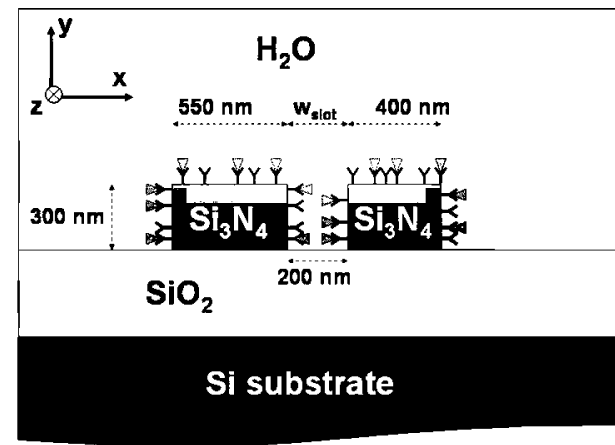

(b)

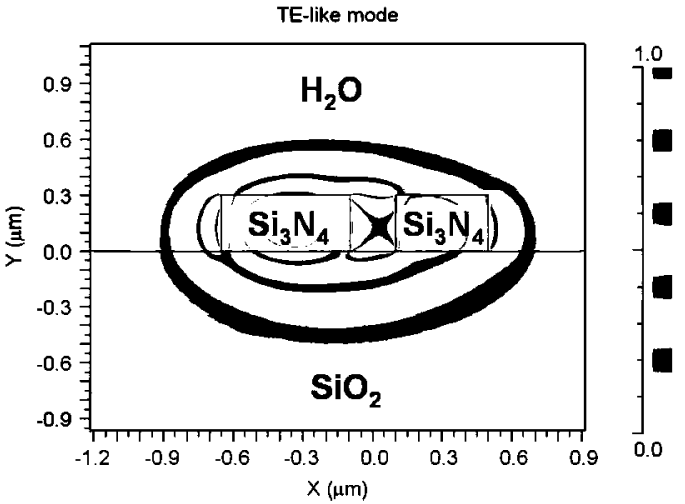

Fig. 1. (a) Schematic cross section of the $\mathrm{Si}_{3} \mathrm{~N}_{4} / \mathrm{SiO}_{2}$ ring slot-waveguide used for optical sensing of biomolecules. Triangles and Y-shaped symbols represent antigen and antibody molecules, respectively. (b) Calculated quasi-TE mode of the ring slot-waveguide turning to the left ( $-x$ axis) with a radius of curvature of $70 \mu \mathrm{m}$ and an operation wavelength of $1.3 \mu \mathrm{m}$. 
shown in Fig. 1(b). The top of the device was opened to expose the ring sensor to the ambient. Fabrication details can be found elsewhere. After fabrication, the chip was cleaned in a $\mathrm{H}_{2} \mathrm{SO}_{4}: \mathrm{H}_{2} \mathrm{O}_{2}$ (3:1) solution to remove organic materials from the sensor surface. After removing the native oxide from the $\mathrm{Si}_{3} \mathrm{~N}_{4}$ sensor surface with highly diluted aqueous HF, the chip was dipped into aqueous glutaraldehyde (5\%) to allow selective, covalent immobilization of anti-BSA antibodies to the $\mathrm{Si}_{3} \mathrm{~N}_{4}$ sensor surface through the formation of imine bonds. Aldehydized surfaces were then incubated for $20 \mathrm{~min}$ into aqueous solutions of anti-BSA (increasing concentrations). Then, surfaces were rinsed with deionized (DI) $-\mathrm{H}_{2} \mathrm{O}$ and blown with $\mathrm{N}_{2}$. Finally, after each anti-BSA incubation/washing step, the sensor was flooded with a drop of DI- $\mathrm{H}_{2} \mathrm{O}$ and optically characterized as described in on a temperature-controlled setup $\left(22^{\circ} \mathrm{C}\right)$.

The described chemical treatment of the sensor chip was first developed and characterized on $\mathrm{Si}_{3} \mathrm{~N}_{4} / \mathrm{SiO}_{2}$ dual-zone planar substrates. Selective functionalization of $\mathrm{Si}_{3} \mathrm{~N}_{4}$ surfaces with glutaraldehyde was demonstrated through hydrophobicity studies of the modified surfaces by using water angle measurements. Then, fluorescence confocal microscopy was employed for both; the immobilization assays with goat antirabbit (GAR) antibodies, and bioactivity immunoassays based on BSA-anti-BSA and streptavidin-biotinylated oligonucleotide systems, using Cy5 molecules as fluorescent labels. These assays showed that antibody immobilization on $\mathrm{Si}_{3} \mathrm{~N}_{4}$ is effective and selective and that the immobilized molecules maintained their activity, allowing the selective capture of the target molecule. The demonstrated bioactivity and selectivity on planar substrates should also occur in the slot-region of a $\mathrm{Si}_{3} \mathrm{~N}_{4}$ slot-waveguide since $w_{\text {slot }}=200 \mathrm{~nm}$ whereas the molecular length scale is in the range of $10 \mathrm{~nm}$.

Figure 2 shows the experimental normalized output power of the slot-waveguide ring resonator for TE-polarization ( $E$ field along the $x$ axis) for different anti-BSA concentrations. It is observed that the reso-

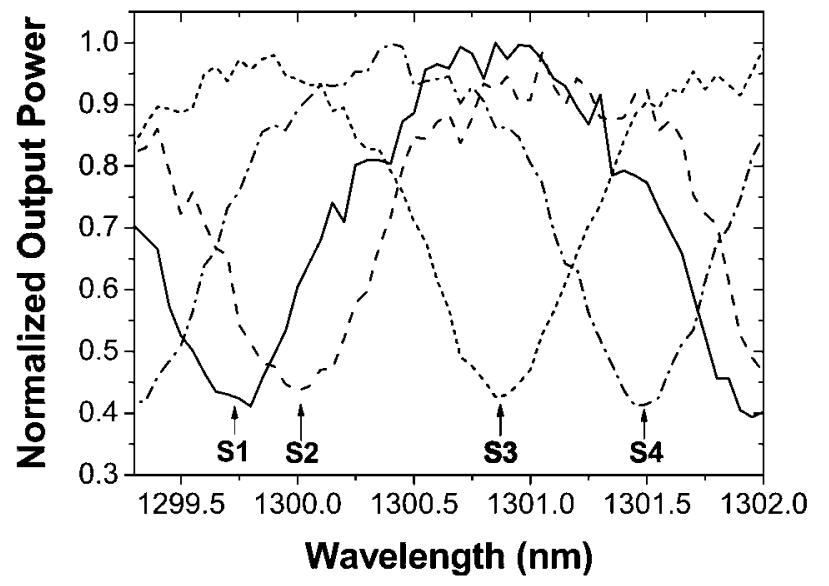

Fig. 2. Normalized transmission spectra of the slotwaveguide microring resonator for TE-polarization for different anti-BSA concentrations: S1 $(0.4 \mu \mathrm{g} / \mathrm{ml}), \quad \mathrm{S} 2$ $(0.44 \mu \mathrm{g} / \mathrm{ml})$, S3 $(2.04 \mu \mathrm{g} / \mathrm{ml})$, and $\mathrm{S} 4(3.64 \mu \mathrm{g} / \mathrm{ml})$. nance wavelength redshifts as the antibody concentration increases. Figure 3 illustrates the resonance wavelength shift as a function of anti-BSA concentration. For anti-BSA concentrations above $17.2 \mu \mathrm{g} / \mathrm{ml}$ the resonance wavelength shift saturates, indicating that the sensor surface is fully covered. This suggests that the device response is solely due to the surface modification since the bulk liquid is pure DI- $\mathrm{H}_{2} \mathrm{O}$ for all measurements. The sensitivity for biomolecule detection is given by $\mathrm{S}_{\mathrm{B}}=\left(\delta \lambda / \sigma_{\mathrm{p}}\right)$, where $\delta \lambda$ is the spectral shift at saturation and $\sigma_{\mathrm{p}}$ is the surface density of a monolayer of biomolecules. As seen in Fig. 3, the saturation spectral shift for anti-BSA detection is $\delta \lambda_{\text {antiBSA }}=3 \mathrm{~nm}$. The surface density of a monolayer of anti-BSA can be calculated from the average length of an anti-BSA molecule, $d_{\text {antiBSA }}=12 \mathrm{~nm}$, as $\sigma_{\mathrm{p}, \text { antiBSA }}=\left(\mathrm{MM}_{\mathrm{antiBSA}} / \mathrm{N}_{\mathrm{A}}\right) /\left(\mathrm{d}_{\text {antiBSA }}\right)^{2}=1.7 \mathrm{ng} / \mathrm{mm}^{2}$, where $\mathrm{MM}_{\text {antiBSA }}=147,000$ is the molecular mass of anti-BSA and $\mathrm{N}_{\mathrm{A}}=6.02 \times 10^{23}$ is Avogadro's number. The calculated value of $\sigma_{\mathrm{p} \text {,antiBSA }}$ is in agreement with experimental values. Thus, the anti-BSA detection sensitivity is $\mathrm{S}_{\text {antiBSA }}=1.8 \mathrm{~nm} /\left(\mathrm{ng} / \mathrm{mm}^{2}\right)$. The biomolecule detection limit is given by $\mathrm{DL}_{\mathrm{B}}$ $=R / S_{B}$, where $R$ is the sensor resolution. In our system setup the sensor resolution is determined by the spectral resolution $(\mathrm{R}=50 \mathrm{pm})$; thus the anti-BSA detection limit will be $\mathrm{DL}_{\text {antiBSA }}=28 \mathrm{pg} / \mathrm{mm}^{2}$. Note that our experimental procedure based on repeated steps is appropriate to analyze the sensor performance based on surface concentration, but it does not allow real-time measurements. This could be achieved by using a flow-injection system

Sensing of BSA-anti-BSA affinity reactions on the device surface was also studied. After saturation of the sensor surface with anti-BSA molecules, a blocking step with ethanolamine was performed to deactivate the remaining aldehyde groups on the surface. The chip was then incubated for $20 \mathrm{~min}$ in aqueous solutions of BSA (increasing concentrations). Then, the surfaces were rinsed with $\mathrm{DI}-\mathrm{H}_{2} \mathrm{O}$ and blown with $\mathrm{N}_{2}$. The sensor, after each incubation/washing step, was exposed to DI- $\mathrm{H}_{2} \mathrm{O}$ and characterized as be-

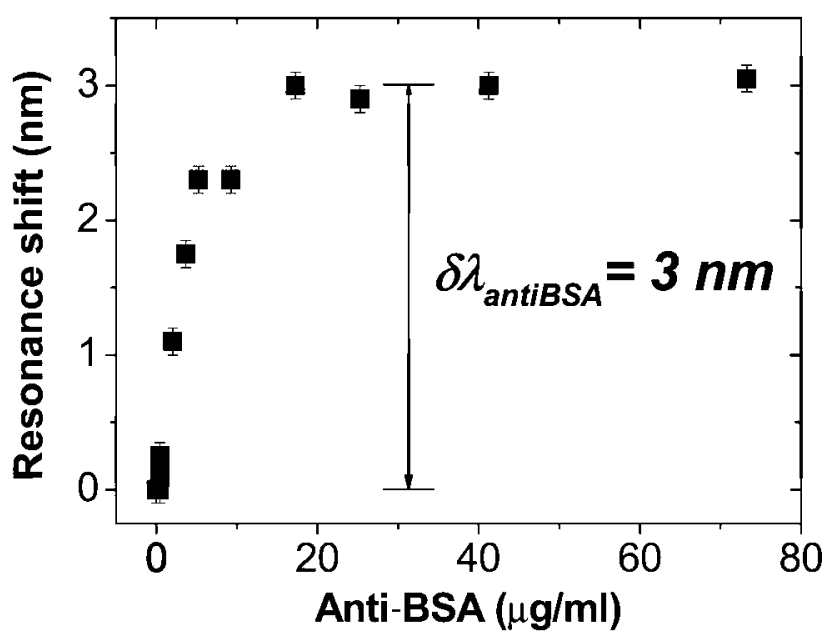

Fig. 3. Resonance wavelength shift versus anti-BSA concentration. $\delta \lambda_{\text {antiBSA }}=3 \mathrm{~nm}$ is the spectral shift at anti-BSA surface saturation. 
fore. Figure 4 shows the measured resonance wavelength shift of the slot-waveguide ring resonator (TEpolarization) for different BSA concentrations. It is observed that the resonance wavelength shift starts to saturate $\sim 5 \mu \mathrm{g} / \mathrm{ml}$, suggesting that BSA molecules only bind to available anti-BSA molecules on the sensor surface until all the binding sites are occupied. For large BSA concentrations, the measured spectral shift deviates from the saturation value and increases with BSA concentration. This can be attributed to an accumulation of BSA molecules by adsorption on the BSA layer bound to the anti-BSA antibodies. The sensitivity for BSA detection $\left(\mathrm{S}_{\mathrm{BSA}}\right)$ can be obtained assuming the same molecular surface density as that for anti-BSA at saturation, and considering that the molecular mass of BSA is MM $_{\mathrm{BSA}}$ $=66,399 \quad \mathrm{|}$. From Fig. 4, the resonance shift at saturation can be considered to be $\delta \lambda_{\mathrm{BSA}}=2.45 \mathrm{~nm}$; thus $\mathrm{S}_{\mathrm{BSA}}=3.2 \mathrm{~nm} /\left(\mathrm{ng} / \mathrm{mm}^{2}\right)$. This leads to a BSA detection limit of $\mathrm{DL}_{\mathrm{BSA}}=16 \mathrm{pg} / \mathrm{mm}^{2}$, limited by wavelength resolution $(\mathrm{R}=50 \mathrm{pm})$. For the sake of comparison, the reported detection limits for biotinylated BSA molecules of a polymer ring biochemical sensor and a Mach-Zehnder interferometer were 250 and $5 \mathrm{pg} / \mathrm{mm}^{2}$, respectively. Recently, singlemolecule detection has been demonstrated by using a microtoroid resonator . The detection limit of that sensor is therefore orders of magnitude smaller than that of our device. However, when comparing these numbers, one should note that there is considerable disparity in interrogation wavelength, resolution, sensor footprint, and functionalized area, the significance of which is application dependent.

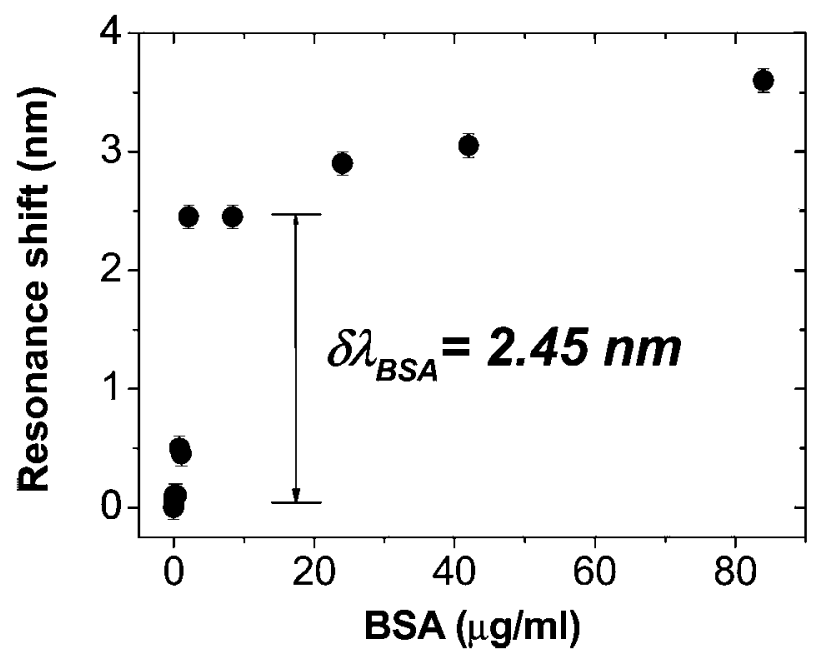

Fig. 4. Resonance wavelength shift versus BSA concentration. $\delta \lambda_{\mathrm{BSA}}=2.45 \mathrm{~nm}$ is the spectral shift at BSA surface saturation.
In conclusion, we have demonstrated highly sensitive molecular binding detection with an integrated $\mathrm{Si}_{3} \mathrm{~N}_{4} / \mathrm{SiO}_{2}$ slot-waveguide microring biosensor. The measurements indicate that the sensor response is solely due to surface modification by molecular binding. The estimated limits of detection of the device for anti-BSA and BSA molecules are 28 and $16 \mathrm{pg} / \mathrm{mm}^{2}$, limited by wavelength resolution. These values favorably compare with those of state-of-the-art integrated photonic biosensors based on conventional waveguides, demonstrating the benefits of using slotwaveguides to detect small surface modifications, such as molecular binding.

C. A. Barrios acknowledges support of the Spanish Ministry of "Educación y Ciencia" (Program "Ramón y Cajal"). K. B. Gylfason acknowledges support of the Steinmaur Foundation, Liechtenstein. This work is done within the FP6-IST-SABIO project (026554), funded by the European Commission and Spanish MEC projects CTQ2007-64735 and TEC200628117-E.

\section{References}

B. J. Luff, J. S. Wilkinson, J. Piehler, U. Hollenbach, J. Ingenhoff, and N. Fabricius, J. Lightwave Technol. 16, 583 (1998).

B. J. Luff, R. D. Harris, and J. S. Wilkinson, Opt. Lett. 21, 618 (1996).

A. Ksendzov and Y. Lin, Opt. Lett. 30, 3344 (2005).

A. Yalçin, K. C. Popat, J. C. Aldridge, T. A Desai, J. Hryniewicz, N. Chbouki, B. E. Little, O. King, V. Van, S. Chu, D. Gill, M. Anthes-Washburn, M. S. Unlü, and B. B. Goldberg, IEEE J. Sel. Top. Quantum Electron. 12, 148 (2006).

C.-Y. Chao, W. Fung, and L. J. Guo, IEEE J. Sel. Top. Quantum Electron. 12, 134 (2006).

A. Schweinsberg, S. Hocdé, N. N. Lepeshkin, R. W. Boyd, C. Chase, and J. E. Fajardo, Sens. Actuators B 123, 727 (2007).

C. A. Barrios, K. B. Gylfason, B. Sanchez, A. Griol, H. Sohlström, M. Holgado, and R. Casquel, Opt. Lett. 32, 3080 (2007).

V. Almeida, Q. Xu, C. A. Barrios, and M. Lipson, Opt. Lett. 29, 1209 (2004).

J. Yang, M. Mayer, J. K. Kriebel, P. Garstecki, and G. M. Whitesides, Angew. Chem., Int. Ed. 43, 1555 (2004). J. P. Ou, S. T. H. Chan, and W. S. B. Yeung, J. Chromatogr., B: Biomed. Appl. 731, 389 (1999).

H.-J. Seok, M.-Y. Hong, Y.-J. Kim, M.-K. Han, D. Lee, J.-H. Leeb, J.-S. Yoob, and H.-S. Kim, Anal. Biochem. 337, 294 (2005).

Swiss-Prot database, Swiss Institute for Bioinformatics (http://us.expasy.org/sprot/sprot-top.html).

A. M. Armani, R. P. Kulkarni, S. E. Fraser, R. C. Flagan, and K. J. Vahala, Science 317, 783 (2007). 